P82 (continued)

Outcome, Measures and Analysis: The evaluation plan included a pre-, post-assessment of parent self-efficacy to engage in healthful practices that promote healthy a healthy child diet. Linear regression models were developed for each outcome variable.

Results: Eleven families enrolled in the study and 10 completed the program. From baseline to final, parents were more confident that they could plan 1 vegetable for lunch and supper $(\mathrm{p}=0.02)$, encourage their child to eat low fat food $(\mathrm{p}=0.02)$, introduce a new vegetable on a monthly basis $(p=0.007)$, keep cut up vegetables in the refrigerator $(\mathrm{p}=0.05)$, and have their child help prepare meals $(\mathrm{p}=0.03)$.

Conclusions and Implications: An innovative nutrition education and cooking class engaged the target audience of parents and preschool-aged children and improved parent confidence level to engage in certain positive parent practices related to improved child diet.

Funding: The Ohio State University Food Innovation Center.

\section{P83 Maternal Compliance to Child Food Requests in Low-income Families}

Amber Haroldson, MS, RD, LDN, arhalvor@uncg.edu, UNC Greensboro, Department of Nutrition, 318 Stone

Building, Greensboro, NC 27407; L. Haldeman, PhD

Objective: The specific aims of this study were to determine foods most often requested by children and frequency of maternal compliance to these requests.

Theory, Prior Research, Rationale: Previous research $(n=135)$ indicated that children play an important role in family dietary decisions. Assessing maternal compliance to child food requests provides insight into the amount of influence a child has on family food consumption.

Study Design, Setting, Participants, and Intervention: Bilingual community interviewers and research assistants conducted 90 minute in-home interviews with low-income mothers and their 7 to 12 year old child. Participants were weighed and measured.

Outcome, Measures and Analysis: Food requests were categorized into 10 food groups (fruits, vegetables, grains, dairy, proteins, fats, sweets, beverages, miscellaneous, combination). Descriptive statistics were conducted to assess compliance.

Results: Forty-four interviews were analyzed (77.3\% Hispanic, 20.5\% African American and 2.3\% Caucasian). The majority of mothers (77.2\%) and children (54.5\%) were overweight/obese. Twenty-three mothers reported child food requests for foods outside of the home and 29 reported requests for foods inside the home on a recent weekday and weekend day. Additionally, 38 mothers reported child food requests while grocery shopping. Mothers reported complying with child food re- quests outside the home (92.6\%), inside the home (73.9\%), and at the grocery store $(65.1 \%)$. Children requested foods in the grain group (crackers, cookies, cereal, pastries) most often, whereas the miscellaneous and fruit groups were least requested. Mothers reported complying with requests in the beverage (sweetened drinks) group most often (88.9\%) and the sweets group least often $(44.1 \%)$.

Conclusions and Implications: Results will be used to target an intervention utilizing child influence in families to promote positive dietary behaviors.

Funding: Southern Rural Development Center, RIDGE Center for Targeted Studies.

\section{P84 Incentivizing Children's Intake of Fruits and Vegetables at School: A U.S. Evaluation of the Food Dudes Program}

Heidi Wengreen, PhD, RD, heidi.wengreen@usu.edu, Utah State University, 8700 Old Main Hill, Logan, UT 84335; S. Aguilar, MS, RD; G. Madden, PhD

Objective: To evaluate the effectiveness of the Food Dudes program designed to increase fruit and vegetable (F/V) consumption among children in U.S. schools.

Theory, Prior Research, Rationale: The Food Dudes program has been successfully implemented in the United Kingdom and other countries, but the efficacy of this program in U.S. schools is unknown.

Study Design, Setting, Participants, and Intervention: Participants were children attending 1 of 6 elementary schools $(n=2440)$. Schools were randomized to receive the full Food Dudes program, a version of the program where tangible rewards were replaced with social praise, or a control condition. Over 16 days, children attending schools assigned to the Food Dudes program watched short videos featuring heroic peers (the $\mathrm{FD}$ ) eating $\mathrm{F} / \mathrm{V}$ and were rewarded for eating $\mathrm{F} / \mathrm{V}$ at lunchtime.

Outcome, Measures and Analysis: F/V consumption was measured by photo analysis 4 times over 4 months. Repeated-measures general linear models were used to assess differences in outcome measures over time.

Results: Average consumption of $\mathrm{F} / \mathrm{V}$ at the baseline assessment was 0.37 cups. Intake increased following the 16-day intervention among children in the intervention schools (by 27-35\%) but not the control schools $(p<0.001)$. The observed increases in $F / V$ intake were not maintained when intake was assessed 3 months later.

Conclusions and Implications: The Food Dudes program when implemented in four U.S. elementary schools resulted in short but not longer-term changes in children's school lunchtime $\mathrm{F} / \mathrm{V}$ consumption. Further work is needed to develop and test strategies that may produce robust, lasting increases in $\mathrm{F} / \mathrm{V}$ consumption among children.

Funding: USDA, Utah Agriculture Experiment Station. 